\title{
Image Transmission: Analog or Digital? ${ }^{\dagger}$
}

\author{
Jose Balsa *, Tomás Domínguez-Bolano, Óscar Fresnedo, José A. García-Naya and Luis Castedo \\ Enxeñaría de Computadores, Facultade de Informática, Universidade da Coruña, Campus de Elviña s/n, \\ 15071 A Coruña, Spain; tomas.bolano@udc.es (T.D.-B.); oscar.fresnedo@udc.es (Ó.F.); \\ jagarcia@udc.es (J.A.G.-N.); luis@udc.es (L.C.) \\ * Correspondence: j.balsa@udc.es \\ + Presented at the XoveTIC Congress, A Coruña, Spain, 27-28 September 2018. \\ Published: 18 September 2018
}

Abstract: Evaluation and comparison of analog and digital wireless transmission systems.

Keywords: analog image transmission; JSCC; image quality comparison; analog image transformation

\section{Introduction}

In this work, we address the design and evaluation of an analog Joint Source Channel Coding (JSCC) system $[1,2]$ for the transmission of grayscale still images. This kind of systems offers some interesting characteristics with respect to digital systems under certain circumstances: high transmission rates over noisy channels and avoiding retransmissions, graceful degradation with a fixed coding scheme (i.e., if the transmitter does not know the quality of the channel) and lower computational complexity.

The second part of this work evaluates the proposed analog system and compares its performance to that of an all-digital system based on JPEG compression. It is very important the selection of the metrics used to evaluate the perceived quality of the received image respect to that of the transmitted one and defining an adequate strategy to make a fair comparison between the analog and digital systems.

\section{System Model}

The transmission system consists of three parts: the transmitter, where the image is prepared, adapted and encoded; the channel; and the receiver, where the image is recovered by two operations: undoing the operations carried out in the transmitter and trying to correct the errors due to the distortions introduced by the wireless channel.

Orthogonal Frequency Division Multiplexing (OFDM) is selected as the waveform for both the analog and the all-digital systems. The latter also encodes the data frames using Turbo Codes. The wireless channel can be (1) a simulated one considering a channel model defined by the ITU or (2) a real one obtained by means of over-the-air transmissions with the GTEC testbed [3-6].

\subsection{Image Pre-Processing}

The image is loaded from a grayscale file with values from 0 to 255 (grayscale intensity). The system divides the image in $8 \times 8$ blocks and they are transformed with a DCT (Discrete Cosine Transform) (Figure 1a) to concentrate most of the image information into a few coefficients (Figure 1b), such that the least relevant coefficients can be disregarded with a small loss in terms of perceived image quality. Each $8 \times 8$ block is further subdivided into four blocks, from the first to the third will be transformed (see Section 2.2), whereas the fourth is always discarded.

After this, the system rearranges the transformed blocks into a sequential data frame (Figure 1c) which is ready for the next step. 


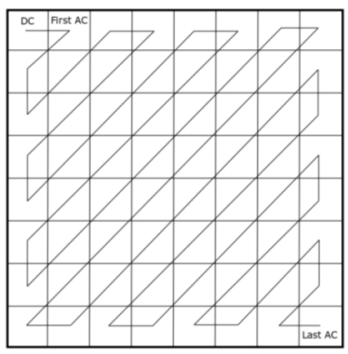

(a)

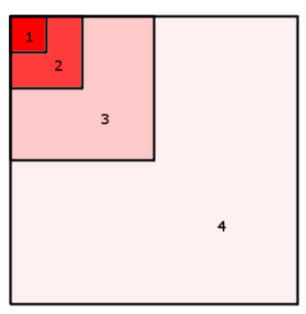

(b)
Data frame - Mapping input

\begin{tabular}{|l|l|l|l|}
\hline 1 & 2 & 3 & 4 \\
\hline
\end{tabular}

Each division includes all sub-block from each and every block in sequence.

(c)

Figure 1. (a) The DCT is applied to each $8 \times 8$ pixel block. (b) Each $8 \times 8$ block is split into 4 sub-blocks in order to apply different analog mapping/transformation schemes. (c) Each sub-block is placed in a sequential data frame.

\subsection{Analog JSCC Scheme}

The system maps the DCT coefficients of the three first blocks (Figure 1c) in channel symbols using analog JSCC techniques [7]. This strategy represents an appealing alternative to the traditional all-digital approaches based on source-channel separation because of its lower complexity and graceful degradation against variations of the channel conditions $[2,8]$. The system works with two analog JSCC methods: expansion and uncoded. The expansion method applies an analog spherical code, based on the exponentially chirped modulation (Equation (1)), whereas the uncoded method performs only a normalization.

$$
x=\Delta\left[\cos (2 \pi \mathbf{s}), \sin (2 \pi \mathbf{s}), \cos (2 \pi \alpha \mathbf{s}), \sin (2 \pi \alpha \mathbf{s}), \ldots, \cos \left(2 \pi \alpha^{\left(\frac{\rho}{2}-1\right)} \mathbf{s}\right), \operatorname{sen}\left(2 \pi \alpha^{\left(\frac{\rho}{2}-1\right)} \mathbf{s}\right)\right]
$$

Equation (1). Analog spherical code used in the encoding operation

s: symbols at the mapping input, $\alpha$ : mapping parameter; $\Delta$ normalization factor; $\rho$ : expansion factor.

At the receiver, we use an optimal Minimum Mean Square Error (MMSE) or a Maximum Likelihood (ML) decoder depending on the selected scheme.

\subsection{Comparison Methodology between Digital and Analog Systems}

The main objective of this work is to compare the transmission speed between the proposed analog system and an all-digital one used as a benchmark. One cannot find in the literature a standard procedure to carry out this very specific comparison. To ensure a fair comparison, we first measure the image quality obtained with the analog system, and next, we design the digital JPEG-based system to compress the source image with that same quality, while ensuring a free-error transmission of the information.

The digital transmission is software-simulated over the estimated channel in the analog system in order to compare the two systems in the same situation. In addition, knowing the channel before transmitting allows for calculating the optimal CQI (Channel Quality Indicator) obtaining the minimal data frame avoiding errors.

The selected quality metric is the SSIM (Structural Similarity Index) [9]. This metric returns a real value 1 (identical images) or less than 1 (different images).

The time needed to transmit the data frames containing the image, encoded with the digital or the analog scheme, is the considered performance metric, which measures the transmission speed.

Acknowledgments: This work has been funded by the Xunta de Galicia (ED431C 2016-045, ED341D R2016/012, and ED431G/01), the Agencia Estatal de Investigacion of Spain (TEC2015-69648-REDC, and TEC2016-75067-C41-R), ERDF funds of the EU (AEI/FEDER, UE), and the predoctoral Grant BES-2014-069772.

Conflicts of Interest: The authors declare no conflict of interest. The founding sponsors had no role in the design of the study; in the collection, analyses, or interpretation of data; in the writing of the manuscript, and in the decision to publish the results. 


\section{References}

1. Ramstad, T.A. Shannon mappings for robust communication. Telektronikk 2002, 98, 114-128.

2. Hekland, F.; Floor, P.A.; Ramstad, T.A. Shannon-Kotel'nikov mappings in joint source-channel coding. IEEE Trans. Commun. 2009, 57, 94-105.

3. Domínguez-Bolaño, T.; Rodríguez-Piñeiro, J.; García-Naya, J.A.; Yin, X.; Castedo, L. Measurement-Based Characterization of Train-to-Infrastructure 2.6 GHz Propagation Channel in a Modern Subway Station. IEEE Access 2018.

4. Domínguez-Bolaño, T.; Rodríguez-Piñeiro, J.; García-Naya, J.A.; Castedo, L. Experimental Characterization of LTE Wireless Links in High-Speed Trains. Wirel. Commun. Mob. Comput. 2017, 5079130, doi:10.1155/2017/5079130.

5. Zhang, L.; Rodríguez-Piñeiro, J.; Fernández, J.R.O.; García-Naya, J.A.; Matolak, D.W.; Briso, C.; Castedo, L. Propagation Modeling for Outdoor-to-Indoor and Indoor-to-Outdoor Wireless Links in High-Speed Train. Measurement 2017, 110, 43-52.

6. Suárez-Casal, P.; Rodríguez-Piñeiro, J.; García-Naya, J.A.; Castedo, L. Experimental Evaluation of the WiMAX Downlink Physical Layer in High-Mobility Scenarios. EURASIP J. Wirel. Commun. Netw. 2015, 109, doi:10.1186/s13638-015-0339-9.

7. Fresnedo, O.; Vazquez-Araujo, F.J.; Castedo, L.; Garcia-Frias, J. Low-complexity near-optimal decoding for analog joint source channel coding using space-filling curves. IEEE Commun. Lett. 2013, 17, 745-748.

8. Vaishampayan, V.A.; Costa, S.I.R. Curves on a sphere, shift-map dynamics, and error control for continuous alphabet sources. IEEE Trans. Inf. Theory 2003, 49, 1658-1672.

9. Wang, Z.; Bovik, A.C. Image Quality Assessment: From Error Visibility to Structural Similarity. IEEE Trans. Image Process. 2004, 13, doi:10.1109/TIP.2003.819861.

(C) 2018 by the authors. Licensee MDPI, Basel, Switzerland. This article is an open access article distributed under the terms and conditions of the Creative Commons Attribution (CC BY) license (http://creativecommons.org/licenses/by/4.0/). 